This is an electronic reprint of the original article. This reprint may differ from the original in pagination and typographic detail.

Author(s): Kuparinen, Liisa; Swan, J. Edward; Rapson, Scott; Sandor, Christian

Title: Depth perception in tablet-based augmented reality at medium- and far-field distances

Year: $\quad 2013$

Version:

Please cite the original version:

Kuparinen, L., Swan, J. E., Rapson, S., \& Sandor, C. (2013). Depth perception in tabletbased augmented reality at medium- and far-field distances. In SAP '13 Proceedings of the ACM Symposium on Applied Perception (pp. 121). Association for Computing Machinery. https://doi.org/10.1145/2492494.2514654

All material supplied via JYX is protected by copyright and other intellectual property rights, and duplication or sale of all or part of any of the repository collections is not permitted, except that material may be duplicated by you for your research use or educational purposes in electronic or print form. You must obtain permission for any other use. Electronic or print copies may not be offered, whether for sale or otherwise to anyone who is not an authorised user. 


\section{Depth Perception in Tablet-Based Augmented Reality at Medium- and Far-Field Distances}

\author{
Liisa Kuparinen \\ University of Jyväskylä \\ Finland \\ liisa.kuparinen@jyu.fi
}

\author{
J. Edward Swan II \\ Mississippi State University \\ USA \\ swan@acm.org
}

\author{
Scott Rapson \\ The University of South Australia \\ Australia \\ scott.rapson@magicvisionlab.com
}

\author{
Christian Sandor \\ The University of South Australia \\ Australia \\ christian.sandor@magicvisionlab.com
}

Current augmented reality (AR) systems often fail to indicate the distance between the user and points of interest in the environment. Empirical evaluations of human depth perception in AR settings compared to real world settings are needed. Our goal in this study was to understand tablet-based AR depth perception by comparing it with real-world depth perception.

Human depth perception has been studied widely for decades. Multiple studies on depth perception in virtual reality (VR) exist, but there are very few which have studied AR. To fill this gap, we replicated and extended a previous study by Lappin et al. [2006], which focused on depth perception in a real world setting. They employed perceptual bisection, in which the observer directs an adjustment person to walk until they are standing halfway between the observer and a target person. They found that observers conduct this task differently depending on the observer's environmental context; they studied an open field, a hallway and a lobby, and had the target person stand either 15 or 30 meters from the observer.

We replicated this task in three contexts: in an open field and a hallway in Australia (Figures 1a and 1b), and on a frozen lake in Finland (Figures 1c-d). In addition to conducting the experiment with an actual target person in the real world (as in Lappin et al. [2006]), we also developed a tablet-based AR application which presented a virtual target person (Figure 1). Furthermore, we varied the presence of feedback about the location of the target person. Observers who saw the virtual target person in the field and hallway did not receive any feedback regarding the target person's distance. In these settings, the adjustment person always began walking next to the observer, and walked towards the target person until directed to stop. However, observers who saw the virtual target person on the lake did receive feedback about the target person's distance. In this setting, during half of the trials the adjustment person started their walk by standing at the same distance (either 15 or 30 meters) as the virtual target person, and walked towards the observer until directed to stop. Furthermore, we told the observers that when the adjustment person stood at these distances, they were standing at the same distance as the virtual target person. On every other trial, this provided feedback about where the virtual target person was standing.

We had a total of 20 observers in the experiments (10 male, 10 female, age range of $22-65$, average 34 years).

The strongest and most consistent result is that both accuracy and precision varied according to the distance of the target. In every experimental situation, we found that the $15 \mathrm{~m}$ result was more accurate, more precise, and slightly overestimated, and that the 30 $\mathrm{m}$ result was less accurate, less precise, and underestimated. In addition, we found a positive effect of feedback at the $30 \mathrm{~m}$ distance, but not at the 15 meter distance. Overall, with feedback, there was no difference between observers' judgments of real and AR targets at $30 \mathrm{~m}$ (average error of $-39 \mathrm{~cm}$ ), but there were differences at $15 \mathrm{~m}$ (average error of $+32 \mathrm{~cm}$ in the real world versus $+103 \mathrm{~cm}$ in AR). In addition, the real-world results failed to replicate several findings of Lappin et al. [2006].

These experiments suggest that, for tablet-based AR applications, results found at one distance will not necessarily predict the results at other distances. In addition, with practice and brief feedback of 20 minutes or less, performance on a depth perceptionrelated task in tablet-based AR approaches real-world performance.

\section{Reference}

LAPPIN, J. S., SHELton, A. L., AND RiESER, J. J. 2006. Environmental context influences visually perceived distance. Perception \& Psychophysics 68, 4, 571-581.

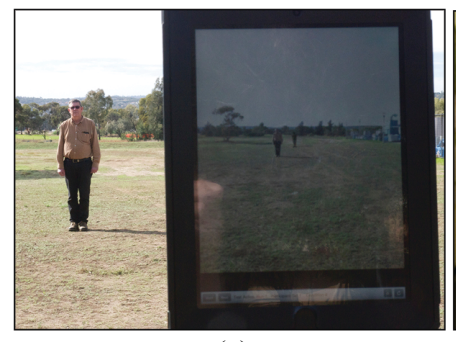

(a)

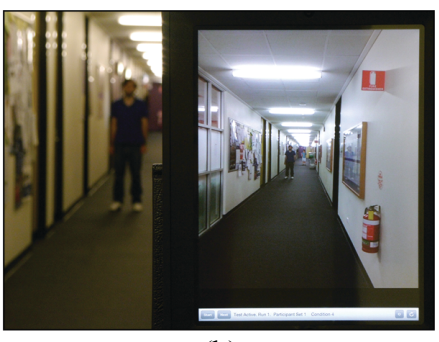

(b)

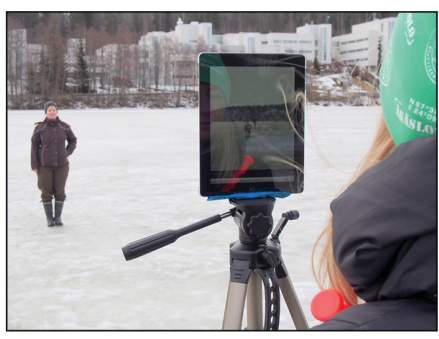

(c)

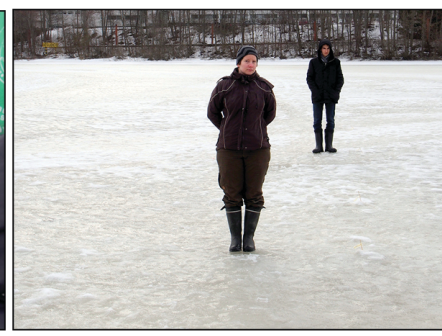

(d)

Figure 1 In our experiments, observers bisected egocentric distances in different contexts by directing an adjustment person towards the middle of the distance to a target person. The target person was either virtual, appearing in an augmented-reality application which ran on a tablet (Figures 1a, 1b, and 1c), or a real person (Figure 1d). Environments included an open field (Figure 1a), a corridor (Figure 1b), and an ice-covered lake (Figures $1 c$ and $1 d$ ). We also varied feedback about the location of the virtual person; in the open field and hallway observers received no feedback, but they did receive feedback on the lake. 